\title{
Ground States of Helium Atom and Hydrogen Negative Ion in the Presence of Magnetic Field Using Variational Monte Carlo Technique
}

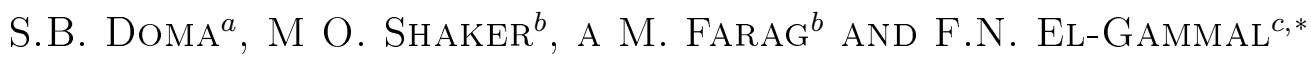 \\ ${ }^{a}$ Mathematics Department, Faculty of Science, Alexandria University, Alexandria, Egypt \\ ${ }^{b}$ Mathematics Department, Faculty of Science, Tanta University, Tanta, Egypt \\ ${ }^{c}$ Mathematics Department, Faculty of Science, Menofia University, Shebin El-Kom, Egypt \\ (Received January 29, 2014; in final form June 15, 2014)
}

Using variational Monte Carlo method, we calculated the $1^{1} 0^{+}$state energies, the derivatives of the total energy and the ionisation energies of the helium atom, and hydrogen negative ion in the presence of magnetic field regime between 0 a.u. and 10 a.u. Our calculations are based on using two types of compact and accurate trial wave functions used before to calculate energies in the absence of magnetic field. Our results are in good agreement with the most recent previous accurate values and also with the exact values.

DOI: $10.12693 /$ APhysPolA.126.700

PACS: 31.10.+z, 31.15.-p, 31.15.V-, 31.15.xt

\section{Introduction}

The problem of the presence of atoms in magnetic fields is a fascinating subject which has attracted a great interest of both experimentalists and theorists in the past three decades. The motivation behind such great interest in this area arises from several sources. In particular, at intermediate field strengths, the nearly equal importance of the Coulomb and magnetic effects has made progress very difficult, and so far only two electron problems have been attempted. This range of field strengths is plagued by the fact that neither the spherical symmetry of the Coulomb potential nor the cylindrical symmetry of the constant magnetic field can be assumed to dominate $[1-4]$.

In contrast to the hydrogen atom, the problem of the helium atom is much more intricate because of the occurrence of the electron-electron repulsion. Even so, many considerable efforts have been devoted to the theoretical investigations of helium atom in intermediate and strong magnetic fields [5-8]. Recently, Wang et al. [9, 10] presented a configuration-interaction (CI) approach which is based on the Hylleraas-Gaussian basis function. Using this method, they introduced a detailed work of the hydrogen atom in magnetic fields and studied the $1^{1} 0^{+}$, $1^{1} 1^{+}$and $1^{1} 2^{+}$states of the helium atom in the magnetic field regime between 0 a.u. and 100 a.u. On the other hand, Zhao et al. adopted a full CI method with Hylleraas-like functions in spherical and cylindrical coordinates to calculate the same states for hydrogen negative ion in strong magnetic fields. Moreover, they calculated the total energies, the derivatives of the total energy with respect to the magnetic field and the ionisation energies, respectively [11, 12]. In Ref. [13], Thirumalai and Heyl calculated the energy levels for both hydrogen and helium atoms in strong magnetic fields as well as the eigenvalues and eigenvectors of the generalized two-dimensional

${ }^{*}$ corresponding author; e-mail: sbdoma@yahoo.com
Hartree-Fock partial differential equations for one- and two-electron systems in a self-consistent manner. They proved that the method is found to be readily extendable to systems with more than two electrons.

Extending to our previous works [14-16], our goal in the present paper is to use the variational Monte Carlo (VMC) method to compute the total energies, the derivatives of the total energy with respect to the magnetic field and the ionisation energies of the helium atom and the hydrogen negative ion in the magnetic field.

\section{Method of calculations}

Quantum Monte Carlo (QMC) methods have proved to be remarkably successful in providing accurate predictions of energies and structures for molecular and atomic systems. These methods are "exact" for systems of a few electrons and highly accurate for systems of thousand of electrons. One of the most important QMC methods is the VMC method which is based on a combination of two ideas namely, the variational principle and the Monte Carlo evaluation of integrals using importance sampling based on the Metropolis algorithm. It is used to compute quantum expectation values of an operator. In particular, if the operator is the Hamiltonian, its expectation value is the variational energy [17],

$$
E_{\mathrm{VMC}}=\int \frac{\left|\Psi_{\mathrm{T}}(\mathcal{R})\right|^{2}}{\left\langle\Psi_{\mathrm{T}} \mid \Psi_{\mathrm{T}}\right\rangle} \frac{\left[\hat{H} \Psi_{\mathrm{T}}\right](\mathcal{R}) d(\mathcal{R})}{\Psi_{\mathrm{T}}(\mathcal{R})},
$$

where $\mathcal{R}=\left(\mathcal{R}_{1}, \mathcal{R}_{2}, \mathcal{R}_{3}, \ldots, \mathcal{R}_{N}\right)$ is a $3 N$-dimensional vector of the electron coordinates. In order to evaluate the integral in Eq. (2.1) we rewrite it as

$$
E_{\mathrm{VMC}}=\int P(\mathcal{R}) E_{L}(\mathcal{R}) \mathrm{d}(\mathcal{R}),
$$

where $P(\mathcal{R})=\frac{\left|\Psi_{\mathrm{T}}(\mathcal{R})\right|^{2}}{\int\left|\Psi_{\mathrm{T}}(\mathcal{R})\right|^{2} \mathrm{~d} \mathcal{R}}$ is positive everywhere and is interpreted as a probability distribution function and $E_{L}(\mathcal{R})=\frac{\left[\hat{H} \Psi_{\mathrm{T}}\right](\mathcal{R})}{\Psi_{\mathrm{T}}(\mathcal{R})}$ is the local energy.

To calculate the integrals in Eq. (2.1) we firstly construct a trial wave function, $\Psi_{\mathrm{T}}^{\alpha}(\mathcal{R})$, depending on a set 
of $\alpha$-variational parameters $\alpha=\left(\alpha_{1}, \alpha_{2}, \ldots \alpha_{N}\right)$ and then vary the parameters to obtain the minimum energy. The value of $E_{L}$ is evaluated using a series of points $\left\{\mathcal{R}_{i}\right\}$ which are sampled from the probability density $P(\mathcal{R})$ using the Metropolis algorithm [18].

In determining the expectation value of the local energy, $\left\langle E_{L}\right\rangle$, it is not necessary to carry out analytic integrations; and, since only differentiation of the trial wave function is required to evaluate the local energy, the trial wave function may take any desired functional form.

At each of the points $\left\{\mathcal{R}_{i}\right\}$, the weighted average $E_{L}(\mathcal{R})$ is evaluated. After a sufficient number of evaluations the VMC estimate of $E_{\mathrm{VMC}}$ will be

$$
E_{\mathrm{VMC}}=\left\langle E_{L}\right\rangle=\lim _{N \rightarrow \infty} \lim _{L \rightarrow \infty} \frac{1}{N} \frac{1}{L} \sum_{j=1}^{N} \sum_{i=1}^{L} E_{L}\left(\mathcal{R}_{i j}\right)
$$

where $L$ is the ensemble size of random numbers $\left\{\boldsymbol{R}_{1}, \boldsymbol{R}_{1}, \ldots, \boldsymbol{R}_{L}\right\}$, which may be generated using a variety of methods [19,20] and $N$ is the number of ensembles. These ensembles so generated must reflect the distribution function itself.

Finally, it is important to calculate the standard deviation of the energy [17]:

$$
\sigma=\sqrt{\frac{\left\langle E_{L}^{2}\right\rangle-\left\langle E_{L}\right\rangle^{2}}{L(N-1)}} .
$$

\section{The trial wave functions}

Our calculations for the ground-state of helium and its isoelectronic ions are based on using two different types of trial wave functions, the first one is a highly compact wave function that has a clear physical meaning and satisfies all the boundary conditions, this wave function is proposed firstly in [21] and is given by

$$
\begin{gathered}
\Psi_{1}\left(r_{1}, r_{2}, r_{12}\right)=(1+\hat{\boldsymbol{P}}) \exp \left(\frac{a_{1} r_{1}+b_{1} r_{1}^{2}}{1+r_{1}}\right) \\
\quad \times \exp \left(\frac{a_{2} r_{2}+b_{2} r_{2}^{2}}{1+r_{2}}\right) \exp \left(\frac{d r_{12}}{1+e r_{12}}\right),
\end{gathered}
$$

where $a_{1}, a_{2}, b_{1}, b_{2}, d$, and $e$ are variational parameters and $\hat{\boldsymbol{P}}$ is the operator that permutes the two electrons. The new feature in this wave function is the functional form: $\exp \left(\frac{a r+b r^{2}}{1+r}\right)$ which helps in satisfying Kato-cusp conditions, which have been stressed in the construction of an accurate wave function previously. This wave function was used to calculate the ground state energy for the He atom and He-like isoelectronic ions for $Z=1$ to 10 and the results obtained were better than pervious works which used compact wave functions for the two-electron systems. The second type of trial wave function takes the form

$$
\begin{aligned}
& \Psi_{2}\left(r_{1}, r_{2}, r_{12}\right)=\left(\frac{2 \beta+1-\mathrm{e}^{-\beta r_{12}}}{2 \beta}\right) \mathrm{e}^{-Z\left(r_{1}+r_{2}\right)} \\
& \quad \times\left[1+C_{1}\left(r_{1}^{2}+r_{2}^{2}\right)+C_{2}\left(r_{1}^{4}+r_{2}^{4}\right)\right]
\end{aligned}
$$

where $\beta, C_{1}$, and $C_{2}$ are variational parameters. $\Psi_{2}$ has been constructed by Rodriguez et al. [22] as a simple function having accuracies and shapes similar to those given by Green et al. [23] or by Chandrasekhar et al. $[24,25]$, but with correct cusp conditions. $\Psi_{2}$ is a product of hydrogenic one-electron solutions and a fully correlated part satisfies all the coalescence cusp conditions at the Coulomb singularities. The application of using $\Psi_{2}$ was extended not only to ground state of helium but also to other ions belonging to the He isoelectronic sequence up to $Z=10$. The results obtained are in good agreement with the exact values.

\section{The Hamiltonian for the two-particle systems in magnetic field}

In our work, we assume that the nuclear mass is infinite and the magnetic field is oriented along the $z$ axis. Hence, the non-relativistic Hamiltonian $H$ for the helium atom and the hydrogen negative ion in a homogeneous magnetic field can be written as [12]:

$$
\begin{aligned}
H & =-\frac{1}{2} \nabla_{1}^{2}-\frac{1}{2} \nabla_{2}^{2}-\frac{Z}{r_{1}}-\frac{Z}{r_{2}}+\frac{1}{r_{12}} \\
& +\left[\frac{\gamma^{2} \rho^{2}}{8}+\frac{\gamma\left(L_{z}+2 S_{z}\right)}{2}\right],
\end{aligned}
$$

where $r_{1}=\left|\boldsymbol{r}_{1}\right|$ and $r_{2}=\left|\boldsymbol{r}_{2}\right|$ in which $\boldsymbol{r}_{1}$ and $\boldsymbol{r}_{2}$ denote the relative radius vectors of the two electrons with respect to the nucleus and $r_{12}=\left|\boldsymbol{r}_{12}\right|$ is the distance between the two electrons, $\rho^{2}=\left(x_{1}^{2}+y_{1}^{2}\right)+\left(x_{2}^{2}+y_{2}^{2}\right), \gamma$ is the magnetic field parameter, $S_{z}$ is the $z$-component of the total spin, $L_{z}$ is the $z$-component of the total angular momentum, $\frac{\gamma^{2} \rho^{2}}{8}$ is the diamagnetic term, $\frac{\gamma}{2} L_{z}$ is the Zeeman term, $-\frac{Z}{r_{1}}-\frac{Z}{r_{2}}$ is the attractive Coulomb interaction with the nucleus (donor) and $\gamma S_{z}$ represents the spin Zeeman term.

Our calculations are based on the Hylleraas coordinates [26], such that $\nabla_{1}^{2}+\nabla_{2}^{2}$ in Eq. (4.1) takes the following form:

$$
\begin{gathered}
\nabla_{1}^{2}+\nabla_{2}^{2}=\frac{\partial^{2}}{\partial r_{1}^{2}}+\frac{2}{r_{1}} \frac{\partial}{\partial r_{1}}+\frac{\partial^{2}}{\partial r_{12}^{2}} \frac{2}{r_{12}} \frac{\partial}{\partial r_{12}} \\
+2 \hat{\boldsymbol{r}}_{1} \hat{\boldsymbol{r}}_{12} \frac{\partial^{2}}{\partial r_{1} \partial r_{12}}+\frac{\partial^{2}}{\partial r_{2}^{2}}+\frac{2}{r_{2}} \frac{\partial}{\partial r_{2}}+\frac{\partial^{2}}{\partial r_{12}^{2}} \\
+\frac{2}{r_{12}} \frac{\partial}{\partial r_{12}}-2 \hat{\boldsymbol{r}}_{2} \hat{\boldsymbol{r}}_{12} \frac{\partial^{2}}{\partial r_{2} \partial r_{12}} \\
\text { 5. Results }
\end{gathered}
$$

The Monte Carlo method described here has been employed for calculating the $1^{1} 0^{+}$state energies of the helium atom in the magnetic field regime between 0 a.u. and 10 a.u. and the hydrogen negative ion from 0 a.u. to 0.8 a.u. All energies are obtained in atomic units, i.e. ( $\hbar=e=m_{e}=1$ ) with a set of $4 \times 10^{7}$ Monte Carlo integration points in order to make the statistical error as low as possible. In the absence of the magnetic field, the parameters appearing in $\Psi_{1}$ were optimized in Ref. [21] and it has been proven that these parameters can be written in a general form depending on the nuclear charge $Z$. Firstly, the two parameters $a_{1}, a_{2}$, and $d$ were fixed $\left(a_{1}=a_{2}=-Z, d=0.5\right)$ in order to satisfy all the cusp conditions. Then, other parameters 
are given by the following relations: $b_{1}=-1.0778 \times Z$, $b_{2}=0.4142-0.8287 \times Z$, and $e=0.2247 \times Z$. Then, the wave function $\Psi_{1}$ exhibits the advantage that it does not need to be optimized each time for a given $Z$ value. Also, for $\Psi_{2}$ we take the values of the variational parameters from Ref. [22] for $\gamma=0$. For every non-zero values of the external magnetic field, the variational parameters appearing in $\Psi_{1}$ and $\Psi_{2}$ were optimized using the steepest descent (SD) method which is considered as one of the most popular methods to optimize the wave function for Monte Carlo methods [27]. We have used SD optimization method successfully in Ref. [28] and accurate results were obtained.

Before presenting the numerical results of our calculations we shall explain our spectroscopic notation in the presence of the field as well as its correspondence to the field-free notation.

According to the four conserved quantities $M, \Pi_{z}, S^{2}$, and $S_{z}$ which characterize the different eigenstates of atoms, we denote a state by $\nu_{S_{z}}^{2 S+1} M(-1)^{\Pi_{z}}$, where $2 S+1$ is the spin multiplicity and $\nu=1,2,3, \ldots$ is the degree of excitation within a given subspace such that $S^{2}$ is the square of the total spin, $\Pi_{z}$ is the $z$-component $\Pi_{z}$ of the total parity, $S_{z}$ is the $z$-component $S_{z}$ of the total spin. For simplicity, we will omit the index $S_{z}$ from this point forward.

In the following we present results for $M=0$ and even $z$-parity. Hence, in the present paper we will investigate and show results for the subspaces $1^{1} 0^{+}$, i.e. $v=1, S=0$ and $M=0$. In the low-field and part of the intermediate regime the $1^{1} 0^{+}$state is the global ground state.

\subsection{The ground state of the helium atom}

For the helium atom $(Z=2)$, we have calculated the total energies and derivatives of the total energy of $1^{1} 0^{+}$ state as functions of the magnetic field. Our calculations for weak magnetic field (0-0.8 a.u.) are based on using the function $\Psi_{1}$, given by Eq. (3.1), where for intermediate range (1-10 a.u.) we used the function $\Psi_{2}$ which is given by $\mathrm{Eq}$. (3.2).

Total energy $E$, electron detachment energy $I$, derivative of the total energy $\frac{\partial E}{\partial \gamma}$ of the $1^{1} 0^{+}$state

TABLE I and one-electron ionisation threshold $T$ of helium atom as functions of the magnetic field strength $\gamma$.

\begin{tabular}{|c|c|c|c|c|c|c|c|c|c|c|}
\hline$\gamma$ & $\begin{array}{l}E\left(1^{1} 0^{+}\right) \\
\text {our work }\end{array}$ & $E^{(8)}$ & $E^{(9)}$ & $E^{(10)}$ & $E^{(11)}$ & $\begin{array}{c}\frac{\partial E}{\partial \gamma} \\
\text { our work }\end{array}$ & $\frac{\partial E}{\partial \gamma}^{(11)}$ & $T$ & $\begin{array}{c}I \\
\text { our work }\end{array}$ & $\begin{array}{c}\text { Standard } \\
\text { deviation } \sigma\end{array}$ \\
\hline 0 & -2.903771 & -2.903724 & -2.903473 & -2.9037155 & -2.903724374 & 0.000 & 0.000 & -2.000 & 0.903771 & $5 \times 10^{-5}$ \\
\hline 0.001 & -2.90378 & & & & -2.903724173 & 0.00397844 & 0.003978101 & -1.999499938 & 0.904280062 & $7 \times 10^{-5}$ \\
\hline 0.005 & -2.903776 & & & & -2.903719398 & 0.01986901 & 0.019869592 & -1.997498438 & 0.906277562 & $2 \times 10^{-4}$ \\
\hline 0.008 & -2.903758 & & & & -2.903711641 & 0.03173584 & 0.031737483 & -1.995996000 & 0.907762 & $4 \times 10^{-4}$ \\
\hline 0.01 & -2.903761 & -2.903704 & -2.903451 & -2.9036898 & -2.903704480 & 0.03963004 & 0.039610410 & -1.994993750 & 0.90876725 & $3 \times 10^{-4}$ \\
\hline 0.02 & -2.903693 & -2.903645 & -2.903386 & -2.9036275 & -2.903644813 & 0.07824952 & 0.078244375 & -1.989975001 & 0.913717999 & $2 \times 10^{-4}$ \\
\hline 0.05 & -2.903288 & & -2.902966 & -2.9032106 & -2.903227357 & 0.18259450 & 0.182410623 & -1.974843777 & 0.928444223 & $4 \times 10^{-4}$ \\
\hline 0.08 & -2.902420 & -2.902453 & & & -2.902453100 & 0.26698340 & 0.266479607 & -1.959600176 & 0.942819824 & $2 \times 10^{-4}$ \\
\hline 0.1 & -2.901723 & -2.901740 & -2.901479 & -2.9017263 & -2.901739559 & 0.00039294 & 0.000397827 & -1.949375430 & 0.95234757 & $5 \times 10^{-4}$ \\
\hline 0.2 & -2.895829 & & -2.895499 & -2.8958159 & -2.895835116 & 0.00079585 & 0.000795654 & -1.897506827 & 0.998322173 & $4 \times 10^{-4}$ \\
\hline 0.5 & -2.856495 & & -2.855906 & -2.8562141 & -2.856237306 & 0.00198963 & 0.001989116 & -1.734628064 & 1.121866936 & $7 \times 10^{-4}$ \\
\hline 0.8 & -2.788485 & -2.788425 & & & -2.788425996 & 0.00318259 & 0.003182532 & -1.561526260 & 1.22695874 & $6 \times 10^{-4}$ \\
\hline 1 & -2.850215 & -2.730373 & -2.730015 & -2.7302745 & -2.7303464 & & 0.312982810 & -1.440989741 & 1.409225259 & $5 \times 10^{-4}$ \\
\hline 2 & -2.307957 & -2.33065 & -2.330270 & -2.3305260 & -2.3306211 & & 0.470160829 & -0.788842154 & 1.519114846 & $6 \times 10^{-4}$ \\
\hline 10 & 3.149141 & & 3.064202 & 3.0636900 & 3.0636148 & & 0.774024780 & 5.609851957 & 2.460710957 & $7 \times 10^{-4}$ \\
\hline
\end{tabular}

${ }^{8}$ total energies obtained from Ref. [8]; ${ }^{9}$ total energies obtained from Ref. [9]; ${ }^{10}$ total energies obtained from Ref. [10]

${ }^{11}$ total energies obtained from Ref. [11]

The derivatives of the total energy with respect to the magnetic field, which can be obtained by the HellmanFeynman theorem [12]:

$$
\frac{\partial E}{\partial \gamma}=\left\langle\left|\frac{\partial H}{\partial \gamma}\right|\right\rangle,
$$

are provided in the seventh column of Table I. The tenth column in Table I provides the values for the identical one-electron ionisation threshold of the state $1^{1} 0^{+}$in terms of the scaling rule [5]:

$$
T(\gamma)=\gamma-4 E(\mathrm{H}, \gamma / 4)
$$

where the binding energy of the ground state of hydro- gen, $E(H, \gamma / 4)$, can be obtained from Ref. [2] and then the one-electron ionisation energies $I(\gamma)$ of $1^{1} 0^{+}$state can be obtained from the one-electron ionisation threshold $T(\gamma)$ by subtracting the corresponding total energies $E(\gamma)$. The obtained results of the state $1^{1} 0^{+}$for the helium atom are presented in Table I with the most accurate available results for comparison. The comparison shows that our results have a good accuracy comparing with the results obtained by other methods. It is seen from Table $I$ that the energy of the $1^{1} 0^{+}$state of the helium atom in the absence of magnetic field is $(-2.903771)$, which is closer to the value $(-2.90372438)$ 
TABLE II

Total energy $E$, electron detachment energy $I$, derivative of the energy $\frac{\partial E}{\partial \gamma}$ of the $1^{1} 0^{+}$state and one-electron ionisation threshold $T$ of hydrogen negative ion as functions of the magnetic field strength $\gamma$.

\begin{tabular}{|c|c|c|c|c|c|c|c|c|c|c|}
\hline$\gamma$ & $\begin{array}{c}E \\
\text { our work }\end{array}$ & $E^{(12)}$ & $\begin{array}{c}I\left(\mathrm{H}^{-}\right) \\
\text {our work }\end{array}$ & $I^{(12)}$ & $I^{(30)}$ & $I^{(31)}$ & $\begin{array}{c}\frac{\partial E}{\partial \gamma} \\
\text { our work }\end{array}$ & $\frac{\partial E}{\partial \gamma}(12)$ & $T$ & $\begin{array}{c}\text { Standard } \\
\text { deviation } \sigma\end{array}$ \\
\hline 0 & -0.5274899 & -0.52774673 & 0.0274899 & 0.02774673 & 0.02754875 & & 0.000 & 0.000 & -0.50000000 & $6 \times 10^{-5}$ \\
\hline 0.0008 & -0.5273412 & -0.52774498 & 0.02774136 & 0.02814514 & 0.02794446 & & 0.00317892 & 0.00317584 & -0.49959984 & $3 \times 10^{-4}$ \\
\hline 0.002 & -0.5279142 & -0.52773712 & 0.0289152 & 0.02873812 & 0.02853877 & 0.02785 & 0.00792587 & 0.00792574 & -0.49899900 & $6 \times 10^{-4}$ \\
\hline 0.005 & -0.5274714 & -0.52769417 & 0.02997765 & 0.03020042 & 0.03000425 & 0.0293 & 0.01975373 & 0.01975019 & -0.49749375 & $2 \times 10^{-4}$ \\
\hline 0.008 & -0.52744414 & -0.52762077 & 0.03146014 & 0.03163677 & 0.03142473 & & 0.03144386 & 0.03144091 & -0.49598400 & $3 \times 10^{-4}$ \\
\hline 0.01 & -0.5274141 & -0.52754683 & 0.0324391 & 0.03257183 & 0.03237472 & 0.0317 & 0.03909503 & 0.03909497 & -0.49497500 & $5 \times 10^{-4}$ \\
\hline 0.02 & -0.5269161 & -0.52697467 & 0.0370157 & 0.03707463 & 0.03687014 & 0.0362 & 0.07536712 & 0.07534053 & -0.4899004 & $2 \times 10^{-4}$ \\
\hline 0.05 & -0.5233312 & -0.52332420 & 0.04895449 & 0.04894749 & 0.04876375 & & 0.16381760 & 0.16328434 & -0.47437671 & $5 \times 10^{-4}$ \\
\hline 0.08 & -0.5173416 & -0.51739859 & 0.0589306 & 0.05898758 & 0.05874669 & & 0.22928120 & 0.22924867 & -0.45841100 & $2 \times 10^{-4}$ \\
\hline 0.1 & -0.5124769 & -0.51244917 & 0.06495042 & 0.06492269 & 0.06470874 & & 0.26525270 & 0.26522837 & -0.44752648 & $3 \times 10^{-4}$ \\
\hline 0.2 & -0.4789198 & -0.47893274 & 0.08853823 & 0.08855118 & 0.08830200 & & 0.394550810 & 0.39446558 & -0.39038157 & $1 \times 10^{-4}$ \\
\hline 0.5 & -0.3285949 & -0.32832661 & 0.13138436 & 0.13111607 & 0.13083820 & & 0.57025990 & 0.58095536 & -0.19721054 & $3 \times 10^{-4}$ \\
\hline 0.8 & -0.1394019 & -0.13965117 & 0.15711851 & 0.15736777 & 0.15710667 & & 0.59094730 & 0.66841077 & 0.01771661 & $5 \times 10^{-4}$ \\
\hline
\end{tabular}

${ }^{12}$ the obtained results of total energy from Ref. [8]; ${ }^{30}$ the obtained results of electron detachment energy from Ref. [30];

31 the obtained results of electron detachment energy from Ref. [31]

obtained by Drake et al. [29]. Figure 1 shows the ionisation energy of the $1^{1} 0^{+}$state obtained with the function $\Psi_{1}$ with respect to the magnetic field strength $\gamma$, for field strengths from $\gamma=0$ to $\gamma=0.8$. Furthermore, Fig. 2 shows the ionisation energy of the state $1^{1} 0^{+}$obtained with the function $\Psi_{2}$ with respect to the magnetic field strength $\gamma$ for field strengths from $\gamma=1$ to $\gamma=10$.

Figures 1, 2 show that the ionisation energies increase monotonically with increasing field strength.

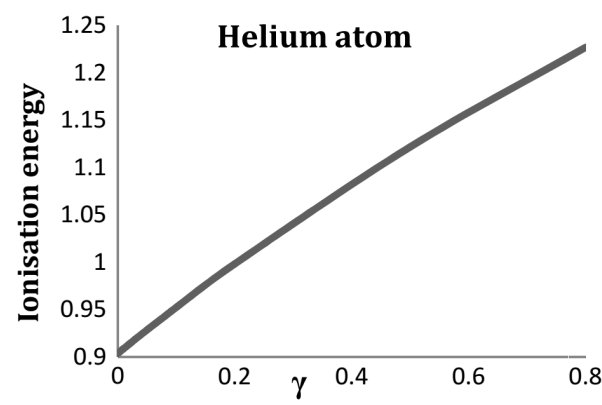

Fig. 1. The ionisation energy of the $1^{1} 0^{+}$state obtained with the function $\Psi_{1}$ versus the magnetic field strength $\gamma$ in a.u.

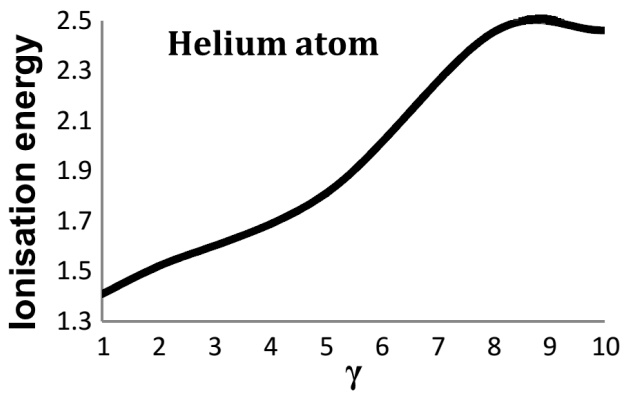

Fig. 2. The ionisation energy of the $1^{1} 0^{+}$state obtained with the function $\Psi_{2}$ versus the magnetic field strength $\gamma$ in a.u.

\subsection{The ground state of the hydrogen negative ion}

The total energies and the electron detachment energies of the state $1^{1} 0^{+}$for the hydrogen negative ion have been calculated as functions of the magnetic field using the function $\Psi_{1}$. Our results are presented in Table II.

The electron detachment energy is defined to be the required energy to remove one electron from the atom without changing the quantum numbers of the total system.

In order to obtain the electron detachment energy, namely one-electron ionization energy

$$
I\left(\mathrm{H}^{-}\right)=T-E,
$$

we have to compute the one-electron ionization threshold

$$
T=\gamma-I(\mathrm{H}),
$$

where $I(\mathrm{H})$, the binding energy of the ground state of hydrogen at magnetic field $\gamma$, is given by Kravchenko et al. [2]. For the $1^{1} 0^{+}$state, the identical one-electron ionization threshold is provided in Table II. In addition, we also calculated the derivatives of the total energy with respect to the magnetic field of the state $1^{1} 0^{+}$, which reflect the changing relationship between the total energies and the field strengths, by means of the HellmanFeynman theorem [12] which is given in Eq. (5.1). We compare our results with those obtained by other methods from $\gamma=0.0$ a.u. to $\gamma=0.8$ a.u. (1.0 a.u. corresponds to $2.35 \times 10^{5} \mathrm{~T}$ ). Our field-free total energy is -0.5274899 , which is closer to the value -0.52775102 calculated by Drake et al. [29] in the Hylleraas coordinates. Besides, it can also be found that the electron detachment energy at zero field obtained by Drake et al. is a little bit higher than ours.

As can be seen from Table II, the $1^{1} 0^{+}$state is raised from -0.5274899 at $\gamma=0.0$ a.u. to -0.1394019 at $\gamma=$ 0.8 a.u. This state is the most tightly bound state for all field strengths.

In addition, the detachment energy increased from 0.0274899 a.u. at $\gamma=0.0$ to 0.15711851 a.u. at $\gamma=0.8$. There are two reasons which give rise to the fact that 


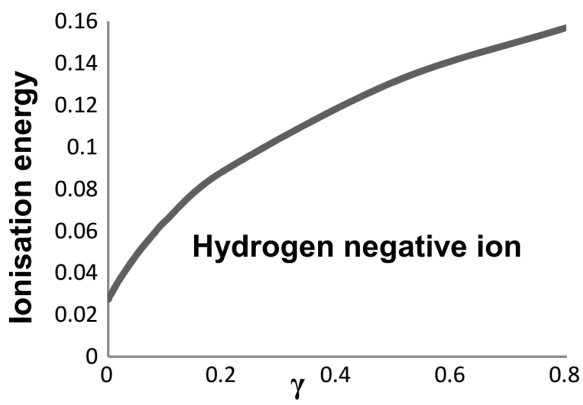

Fig. 3. The ionisation energy of the $1^{1} 0^{+}$state obtained with the function $\Psi_{1}$ versus the magnetic field strength $\gamma$ in a.u.

this state is the most tightly bound one. On the one hand, the electrons are in this state much closer to the nucleus than in other states. This increases the binding due to the attractive nuclear potential energy. On the other hand, correlation has an important impact on the binding energy. Both effects are reinforced with increasing field strength as the electrons become more and more confined in the $x-y$ plane perpendicular to the magnetic field. These effects overcome the influence of the static electron-electron repulsion. It is clear that our results are in good agreement with the previous data. Figure 3 shows that the ionisation energy of the state $1^{1} 0^{+}$increases with respect to the magnetic field strength $\gamma$ for field strengths from $\gamma=0$ to $\gamma=0.8$.

\section{Conclusions}

In the present work, we have studied the helium atom and the hydrogen negative ion in the presence of a magnetic field by using the well known variational Monte Carlo method. Accordingly, we have calculated the total energies, the derivatives of the total energy with respect to the magnetic field and the ionisation energies of the $1^{1} 0^{+}$state for both the helium and the hydrogen negative ions by using two accurate trial wave functions. High accuracy results are obtained in the low and the intermediate fields due to the fact that we have used two trial wave functions, each of them takes into consideration the electron-electron correlation. Our results are in good agreement with the most recent previous accurate values.

\section{Acknowledgments}

The authors are every grateful to the reviewer for his valuable comments and directions.

\section{References}

[1] J.F. Rivas-Silva, Int. J. Quantum Chem. 51, 135 (1994).

[2] Y.P. Kravchenko, M.A. Liberman, B. Johansson, Phys. Rev. A 54, 287 (1996).

[3] Y.P. Kravchenko, M.A. Liberman, B. Johansson, Phys. Rev. Lett. 77, 619 (1996).
[4] L.B. Zhao, M.L. Du, Commun. Theor. Phys. 52, 339 (2009).

[5] W. Becken, P. Schmelcher, F.K. Diakonos, J. Phys. B, At. Mol. Opt. Phys. 32, 1557 (1999).

[6] W. Becken, P. Schmelcher, J. Phys. B, At. Mol. Opt. Phys. 33, 545 (2000).

[7] W. Becken, P. Schmelcher, Phys. Rev. A 63, 053412 (2001).

[8] M. Hesse, D. Baye, J. Phys. B, At. Mol. Opt. Phys. 37, 3937 (2004).

[9] X.F. Wang, H.X. Qiao, Phys. Rev. A 77, 043414 (2008).

[10] X.F. Wang, J.J. Zhao, H.X. Qiao, Phys. Rev. A 80 , 053425 (2009).

[11] J.J. Zhao, X.F. Wang, H.X. Qiao, Chin. Phys. B 19 113102 (2010).

[12] J.J. Zhao, X.F. Wang, H.X. Qiao, Chin. Phys. B 20 , 053101 (2011).

[13] A. Thirumalai, J.S. Heyl, Phys. Rev. A 79, 012514 (2009).

[14] S.B. Doma, F.N. El-Gammal, Appl. Math. Inf. Sci. 5, 315S (2011).

[15] S.B. Doma, F.N. El-Gammal, Acta Phys. Pol. A 122, 42 (2012)

[16] S.B. Doma, F.N. El-Gammal, J. Theor. Appl. Phys. 6, 28 (2012).

[17] S. Pottorf, A. Puzer, M.Y. Chou, Eur. J. Phys. 20, 205 (1999).

[18] N. Metropolis, A.W. Rosenbluth, N.M. Rosenbluth, A.M. Teller, E. Teller, J. Chem. Phys. 21, 1087 (1953).

[19] R.W. Hamming, Numerical Methods for Scientists and Engineers, 2nd ed., McGraw-Hill, New York 1973.

[20] A. Papoulis, Probability, Random Variables, and Stochastic Processes, McGraw-Hill, New York 1965.

[21] D. Bressanini, G. Morosi, J. Phys. B, At. Mol. Opt. Phys. 41, 145001 (2008).

[22] K.V. Rodriguez, G. Gasaneo, D.M. Mitnik, J. Phys. B, At. Mol. Opt. Phys. 40, 3923 (2007).

[23] L.C. Green, M.M. Mulder, P.C. Milner, Phys. Rev. 91, 35 (1953).

[24] S. Chandrasekhar, D. Elbert, G. Herzberg, Phys. Rev. 91, 1172 (1953).

[25] S. Chandrasekhar, G. Herzberg, Phys. Rev. 98, 1050 (1955).

[26] M.B. Ruiz, Int. J. Quantum Chem. 101, 246 (2005).

[27] M.E. Foulaadvand, M. Zarenia, Int. J. Mod. Phys. C 21, 523 (2010).

[28] F.N. El-Gammal, Alex. J. Math. 2, 1 (2011).

[29] G.W.F. Drake, M.M. Cassar, R.A. Nistor, Phys. Rev. A 65, 054501 (2002).

[30] O.A. Al-Hujaj, P. Schmelcher, Phys. Rev. A 61, 063413 (2000).

[31] C.H. Park, A.F. Starace, Phys. Rev. A 29, 442 (1984). 